\title{
Foliation-dependence of CFTs in Lorentzian-AdS/CFT
}

\author{
Borun D. Chowdhury ${ }^{1, *}$ and Maulik K. Parikh ${ }^{1,2, \dagger}$ \\ ${ }^{1}$ Department of Physics, Arizona State University, Tempe, Arizona 85287, USA \\ ${ }^{2}$ Beyond Center for Fundamental Concepts in Science, Arizona State University, \\ Tempe, Arizona 85287, USA \\ (Received 6 November 2015; published 23 February 2016)
}

\begin{abstract}
In the Lorentzian AdS/CFT correspondence, CFTs are identified by asymptotic boundary surfaces and the boundary conditions imposed on those surfaces. However, AdS can be foliated in various ways to give different boundaries. We show that the CFTs obtained using certain distinct foliations are different. This happens because the asymptotic region of a foliation overlaps with the deep interior region of another. In particular, we focus on the CFTs defined on surfaces of large constant radius in global coordinates, RindlerAdS coordinates, and Poincaré coordinates for $\mathrm{AdS}_{3}$. We refer to these as global-CFT, Rindler-CFT and Poincare-CFT, respectively. We demonstrate that the correlators for these CFTs are different and argue that the bulk duals to these should agree up to very close to the respective horizons but then start differing. Since the BTZ black hole is obtained as a quotient of $\mathrm{AdS}_{3}$, we discuss the implications of our results for bulk duals of periodically identified Poincaré and Rindler-CFTs. Our results are consistent with some recent proposals suggesting a modification of the semiclassical BTZ geometry close to the horizons.
\end{abstract}

DOI: 10.1103/PhysRevD.93.046004

\section{INTRODUCTION}

In Lorentzian AdS/CFT, the definition of a dual CFT involves the conformal boundary surface on which it is supposed to "live" [1] and the boundary conditions on that surface [2-5]. However, different foliations of AdS result in different boundaries [6-8]. For example, one definition of a boundary is on a surface of large radius in global coordinates, another is on a surface of large radius in Poincaré coordinates and yet another is on a surface of large radius in Rindler-AdS coordinates. In this paper, we point out an interesting subtlety of Lorentzian AdS/CFT. Fields in Lorentzian AdS have both normalizable and nonnormalizable modes. Here we show that switching between different foliations of spacetime results in a mixing between these. In particular, for the metric, different foliations mix the $g_{a b}^{(0)}$ and $g_{a b}^{(2)}$ pieces in the Fefferman-Graham expansion. Since normalizable and non-normalizable modes correspond to physical states and sources in the dual CFT, this would presumably correspond to a mixing between these. We regard the corresponding CFTs for these different foliations as "different" (loosely speaking) in the above sense. We refer to these CFTs as the global-CFT, the Poincaré-CFT and the Rindler-CFT, respectively. For simplicity, we discuss only $\mathrm{AdS}_{3}$ which is also easiest to

\footnotetext{
*bdchowdh@asu.edu

†maulik.parikh@asu.edu
}

Published by the American Physical Society under the terms of the Creative Commons Attribution 3.0 License. Further distribution of this work must maintain attribution to the author(s) and the published article's title, journal citation, and DOI. visualize, but many of the results are generalizable to higher dimensions.

There is a quick way to see this. A CFT is defined on a constant radial surface by performing a Fefferman-Graham [9] expansion for some foliation, keeping the leading term fixed and letting the subleading terms fluctuate [1]. It turns out that every large global radius surface invariably intersects surfaces of arbitrarily small Rindler-AdS radius [7] (see Fig. 1). Thus, when defining the global-CFT one invariably ends up imposing conditions on subleading terms in the Fefferman-Graham expansion for the Rindler-AdS foliation. Moreover, in some ranges of parameters, the Fefferman-Graham expansion itself breaks down due to a small Rindler-AdS radius. Conversely, defining the Rindler-CFT one does not impose any conditions on the global boundary outside a finite domain. Similar arguments hold for the Poincaré-CFT.

Bulk horizons projected onto the global boundary give the edges of the so-called causal diamonds. In Sec. II we demonstrate the mismatch of Fefferman-Graham expansions between various foliations at the edges of the causal diamonds. The width of the mismatch region is controlled by the UV cutoff. We also demonstrate how, in the vicinity of the center of the causal diamonds, the various CFTs can be viewed as conformally related. However, it should be noted that due to the incompatibility of Fefferman-Graham expansions at the edges of the causal diamond, the various CFTs cannot be globally related by conformal transformations and are thus not truly equivalent.

In Sec. III we restrict the global CFT to be within the causal diamond by focusing on causal developments of subregions; we refer to these as Rindlerized-global-CFTs and Poincarized-global-CFTs. Correlators within these can 
be analytically continued to the entire global boundary cylinder. We demonstrate how the correlators of Poincarized-global-CFTs (Rindlerized-global-CFTs) are approximately the same as those of Poincaré-CFTs (Rindler-CFTs) deep inside the causal diamonds but differ at the edges. We regard this as evidence that, generically, the correlators of Poincaré-CFT (Rindler-CFT) cannot be analytically continued to outside the causal diamonds.

In Sec. III C we expound on how the CFTs are different, in the above sense. We argue that seen as part of the globalCFTs, there is an interaction between the Hilbert spaces associated with the Rindlerized-global-CFTs whereas the Hilbert spaces associated with the Rindler-CFTs are not interacting. Thus, it may be possible to view the RindlerCFTs as a deformation of the global-CFTs which breaks the concerned interaction. Similar ideas apply for the PoincaréCFTs. We hope to come back to this issue in the future.

In Sec. IV we investigate the implications for the bulk physics. Since the correlators of these CFTs are different, the bulk duals to these CFTs should be different also. As the CFTs differ at the edges of the causal diamonds, and since the causal diamonds are projections of bulk horizons onto the global boundary, we conjecture that the bulk dual of Rindler-CFTs and Poincaré-CFTs should resemble semiclassical global AdS until very close to the respective horizons and then start differing. The width of the transition region is governed by the UV cutoff.

This result is particularly interesting in the context of $\mathrm{AdS}_{3}$, where it becomes relevant to black holes. The BTZ black hole [10] can be viewed as a quotient of $\mathrm{AdS}_{3}$ space [11]. The massless and massive ones come from foliating in Poincaré and Rindler-AdS coordinates, respectively, and periodically identifying along a spatial isometry. The massless BTZ black hole has a singular horizon because of vanishing size so the region behind the horizon is not accessible in supergravity. However, for the massive BTZ black hole the identification produces orbifold singularities (interpreted as the eternal black hole singularity) behind the horizons (interpreted as the eternal black hole event horizons) but is innocuous on the horizons themselves. Thus, one may be inclined to think that dynamics involving horizon crossing in global $\mathrm{AdS}_{3}$ might carry over trivially to BTZ. One may further be inclined to think that such dynamics and, more generally, the interior of the BTZ may be captured by quotients of global-CFTs [12-15].

However, the discrete symmetries relevant for orbifolding to obtain the BTZ black holes are isometries of constant Poincaré and Rindler-AdS radial surfaces and not of surfaces of constant global radius. Thus a natural question is as follows: What are the bulk duals to periodically identified Poincaré-CFTs (PIPCs) and periodically identified Rindler-CFTs (PIRCs)? The dynamics of the quotiented bulk duals to global-CFTs (i.e. the BTZ black holes) cannot be trivially assumed to give the dynamics of the bulk duals to PIPCs and PIRCs. After periodic identification the edges of the causal diamonds correspond to large times, so our results indicate that the correlators of PIPCs and PIRCs differ from the naive ones found from the BTZ geometries at late times [13]. Furthermore, our conjecture implies that bulk duals to these CFTs will resemble the massless and massive BTZ, respectively, until very close to the horizon and then start differing.

In Sec. IV B we focus on the massless BTZ black hole. In the case of the D1-D5 system the near-horizon naive geometry is the massless BTZ $\times S^{3} \times T^{4}$ and the actual geometries are the Lunin-Mathur geometries. The typical ones resemble the massless BTZ black hole until very close to the "would-be" horizon and then start differing. In addition, the PIPC correlators dual to these geometries show the late-time deviations from the naive ones. So in hindsight, the ideas stated in the previous paragraph have already been realized for this case.

In Sec. IV C we focus on the massive BTZ black hole. The story for the massive BTZ black hole is not as well settled as for the massless case. Recently Ref. [16] claimed that the bulk is unstable to small fluctuations. Further, problems related to the holographic relation between the bulk and boundary proposed in [14] (see also [17]) have been raised in [18-20]. In fact, Refs. [16,19,20] have made conjectures which amount to claiming that the dual to PIRCs have the regions behind the horizons removed and end in capped (quantum) geometries beyond the would-be horizons (see also [21,22]). Our results are consistent with the proposals of $[16,19,20]$ and raise further issues with the proposal of [14].

\section{DIFFERENT BOUNDARIES AND DIFFERENT CFTS}

\section{A. Boundary CFT}

To equate the dynamics in AdS to those in a CFT, one needs the so-called dictionary between them. The first entry in this dictionary is the definition of the "boundary" on which the CFT is supposed to live (loosely speaking, since AdS and CFT are dual descriptions). Asymptotically AdS spacetimes admit a Fefferman-Graham expansion of their metrics:

$$
d s^{2} \rightarrow \frac{d r^{2}}{r^{2}}+\left(r^{2} g_{a b}^{(0)}+g_{a b}^{(2)}+\mathcal{O}\left(r^{-2}\right)\right) d x^{a} d x^{b} .
$$

The boundary is understood to be at a fixed large value of $r$ that we refer to as $r_{c}$. This location is related to the cutoff of the dual theory, and a CFT is obtained by taking $r_{c} \rightarrow \infty$. The coordinates $x^{a}$ span the field theory directions. Since the metric blows up for large $r$, the metric on AdS does not define a metric on the boundary but instead yields a conformal structure. Thus, $g_{a b}^{(0)}$ is the boundary metric up to Weyl transformations [23]. The on-shell variation of the gravity action, which includes the Einstein-Hilbert term, the Gibbons-Hawking term, and a divergence-cancelling counterterm [1], 


$$
\begin{aligned}
S= & \frac{1}{16 \pi G} \int d^{d+1} x \sqrt{g^{(d+1)}}(R-2 \Lambda) \\
& +\frac{1}{8 \pi G} \int_{\partial M} d^{d} x \sqrt{g^{(d)}} K+\frac{1}{8 \pi G} S_{c t}\left(g^{(d)}\right),
\end{aligned}
$$

gives

$$
\delta S=\frac{1}{2} \int_{\partial M} d^{2} x \sqrt{-g^{(0)}} T^{a b} \delta g_{a b}^{(0)},
$$

where $T^{a b}$ is a symmetric tensor that is interpreted as the expectation value of the stress tensor of the CFT [1]. The variational principle is well posed if we impose Dirichlet boundary conditions $\delta g_{a b}^{(0)}=0 .^{1}$

Imposing a boundary condition specifies the theory; Dirichlet boundary conditions, in particular, amount to "holding the boundary fixed" [4]. Here, $g_{a b}^{(2)}$ is allowed to fluctuate and captures information of the state. In fact for flat boundaries, $T^{a b} \sim g_{a b}^{(2)}$, and this information is thus encoded in the stress tensor.

Implicit in the choice of boundary conditions is the choice of surface on which such conditions are being imposed. Equation (1) does not allow such a choice because the choice has already been made by foliating spacetime in a particular way. We will discuss more about this issue of choice of boundary surfaces below.

\section{B. States in the boundary CFT}

In the special case of $\mathrm{AdS}_{3}$ we have another way to understand the boundary conditions. Brown and Henneaux [24] have shown that diffeomorphisms with the asymptotic (large $r$ ) form

$$
\begin{gathered}
x^{+} \rightarrow x^{+}-\xi^{+}-\frac{1}{2 r^{2}} \partial_{-}^{2} \xi^{-}, \\
x^{-} \rightarrow x^{-}-\xi^{-}-\frac{1}{2 r^{2}} \partial_{+}^{2} \xi^{+}, \\
r \rightarrow r+\frac{r}{2}\left(\partial_{+} \xi^{+}+\partial_{-} \xi^{-}\right),
\end{gathered}
$$

where $x^{ \pm}=t \pm x$, preserve the asymptotic boundary conditions

$$
\begin{gathered}
g_{+-}=-\frac{r^{2}}{2}+\mathcal{O}(1), \quad g_{++}=\mathcal{O}(1), \quad g_{--}=\mathcal{O}(1), \\
g_{r r}=\frac{1}{r^{2}}+\mathcal{O}\left(r^{-4}\right), \quad g_{+r}=\mathcal{O}\left(r^{-3}\right), \quad g_{-r}=\mathcal{O}\left(r^{-3}\right) .
\end{gathered}
$$

In the $r \rightarrow \infty$ limit the transformations of $x^{ \pm}$induce conformal transformations on the boundary CFT, and this

\footnotetext{
${ }^{1}$ Certain other boundary conditions are also allowed [3-5], but for simplicity we only discuss Dirichlet boundary conditions.
}

FIG. 1. The boundary of AdS for global foliation is shown in gray. This is a surface of constant radius in global coordinates for large values of the radius. The yellow surfaces are surfaces of constant Rindler-AdS radius. It is easy to see that fixing boundary conditions on the global boundary imposes conditions on small Rindler-AdS radial surfaces also. Conversely, putting boundary conditions on just large Rindler-AdS radial surfaces does not put any boundary conditions on the global boundary outside a finite domain. Thus, the global-CFT and the Rindler-CFT are different and imply different dynamics for the bulk.

is reflected in changes in $g^{(2)}$ while at the same time keeping $\delta g^{(0)}=0$.

We see from (6) that conformal transformations inducing diffeomorphisms change the location of the boundary surface. So different foliations permitting asymptotic Fefferman-Graham forms do not immediately imply that the associated CFTs are genuinely different. In particular, they are not different if they are related by a BrownHenneaux transformation as then there is a conformal mapping between the two. ${ }^{2}$

\section{Global vs Rindler boundary}

\section{Global and Rindler-AdS foliations and their boundaries}

One can write the global $\mathrm{AdS}_{3}$ line element as ${ }^{3}$

$$
d s^{2}=\frac{d \rho^{2}}{\rho^{2}+1}-\left(\rho^{2}+1\right) d \tau^{2}+\rho^{2} d \phi^{2}
$$

\footnotetext{
${ }^{2}$ We thank Nemani Suryanarayana for discussions on this point.

${ }^{3}$ For definiteness we discuss only three-dimensional AdS. The results can be generalized to higher dimensions in a straightforward way for most of the paper. An exception is the discussion of the BTZ black hole which can be viewed as a quotient of $\mathrm{AdS}_{3}$; higher-dimensional eternal AdS black holes cannot be obtained as quotients of $\mathrm{AdS}$.
} 


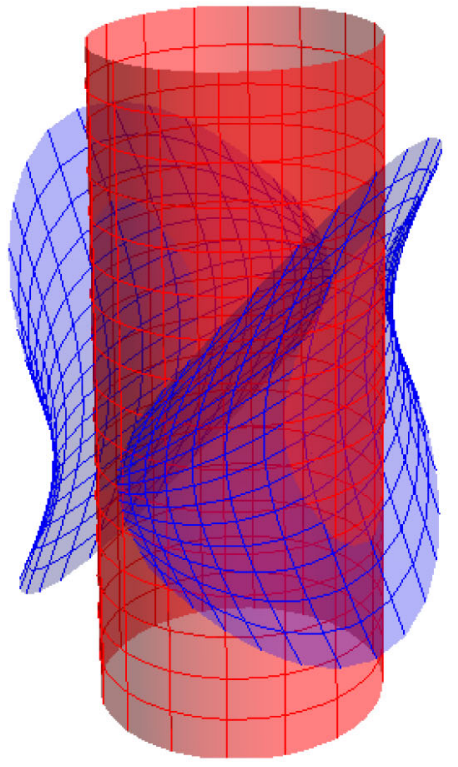

(a) Finite cutoff surfaces

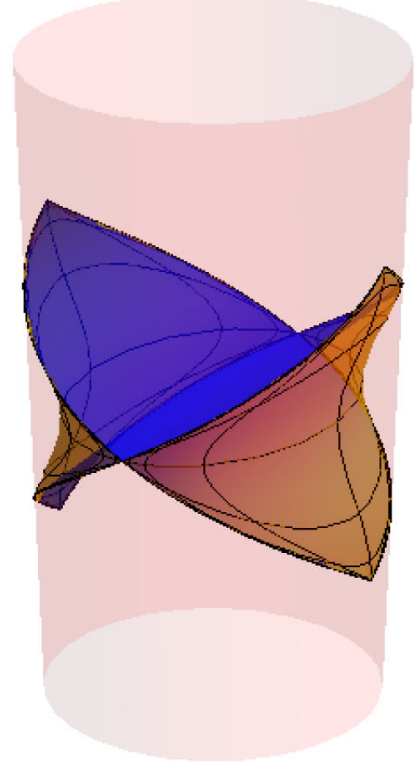

(b) Infinite cutoff surfaces

FIG. 2. (a) The global cutoff surface $\rho=\rho_{c}$ is shown in red, and the Rindler cutoff surface $r_{\mathrm{R}}=r_{\mathrm{R} c}$ is shown in blue. (b) When we take $\rho_{c}$ to infinity, all the $r_{\mathrm{R}}$ surfaces bunch up along the edges of the "causal diamond." Two of them are shown in the figure.

where $\rho \in[0, \infty), \tau \in(-\infty, \infty)$ and $\phi \sim \phi+2 \pi .{ }^{4}$ These coordinates cover the entire manifold. It is often useful to conformally compactify $\rho$ and visualize $\mathrm{AdS}_{3}$ as a solid cylinder (Fig. 1). Similarly, one can also write the metric for the Rindler-AdS wedges in BTZ form:

$$
d s^{2}=\frac{d r_{\mathrm{R}}^{2}}{r_{\mathrm{R}}^{2}-1}-\left(r_{\mathrm{R}}^{2}-1\right) d t_{\mathrm{R}}^{2}+r_{\mathrm{R}}^{2} d x_{\mathrm{R}}^{2}
$$

where $r_{\mathrm{R}} \in(1, \infty), t_{\mathrm{R}} \in(-\infty, \infty)$ and $x_{\mathrm{R}} \in(-\infty, \infty)$. There is an acceleration horizon at $r_{\mathrm{R}}=1$ and these coordinates cover the region outside the horizon [6,25-28]. The rest of $\mathrm{AdS}_{3}$ may be viewed as a Kruskallike extension of these coordinates. The temperature associated with the acceleration horizon can be read off by Wick rotation, and demanding the absence of a conical singularity, it turns out to be $\frac{1}{2 \pi}{ }^{5}$

One can perform a large $\rho$ expansion to write (8) in the Fefferman-Graham form [9] and define a CFT on the cylindrical boundary $S^{1} \times R$ at $\rho=\rho_{c} \rightarrow \infty$. One can also perform a large $r_{R}$ expansion to write (9) in the Fefferman-Graham form and define two CFTs on $R^{1,1} \times R^{1,1}$ at $r_{\mathrm{R}}=r_{\mathrm{R} c} \rightarrow \infty$. ${ }^{6}$ We refer to the former

\footnotetext{
${ }^{4}$ All throughout this paper we set the AdS radius to unity.

${ }^{5}$ There are ways to foliate AdS to give an inner horizon as well $[15,29]$ that corresponds to a rotating BTZ string. For simplicity we will not discuss those.

${ }^{6}$ Care must be taken to redefine the radial coordinates to put the metric in the Fefferman-Graham form to read off the values of $g_{a b}^{(0)}$ and $g_{a b}^{(2)}$.
}

CFT as the global-CFT and the latter CFT pair as the Rindler-CFTs.

It has been claimed that these two CFTs are equivalent (see $[2,30]$, for example). We will argue that this is not the case.

Let us begin by writing down the expressions relating global to Rindler-AdS coordinates:

$$
\begin{gathered}
\rho^{2}=\left(r_{\mathrm{R}}^{2}-1\right) \frac{\cosh 2 x_{\mathrm{R}}+\cosh 2 t_{\mathrm{R}}}{2}+\sinh ^{2} x_{\mathrm{R}}, \\
\cot \phi=-\frac{r_{\mathrm{R}}}{\sqrt{r_{\mathrm{R}}^{2}-1}} \frac{\sinh x_{\mathrm{R}}}{\cosh t_{\mathrm{R}}} \\
\tan \tau=\frac{\sqrt{r_{\mathrm{R}}^{2}-1}}{r_{\mathrm{R}}} \frac{\sinh t_{\mathrm{R}}}{\cosh x_{\mathrm{R}}}
\end{gathered}
$$

and the inverse relations

$$
\begin{gathered}
r_{\mathrm{R}}^{2}-1=-\left(\rho^{2}+1\right) \sin ^{2} \tau+\rho^{2} \sin ^{2} \phi, \\
\tanh x_{\mathrm{R}}=-\frac{\rho}{\sqrt{\rho^{2}+1}} \frac{\cos \phi}{\cos \tau}, \\
\tanh t_{\mathrm{R}}=\frac{\sqrt{\rho^{2}+1}}{\rho} \frac{\sin \tau}{\sin \phi} .
\end{gathered}
$$

From (13) it is clear that for any given $\rho_{c}$, one gets $r_{\mathrm{R}}=1$ (the Rindler-AdS horizon) for suitable values of $\phi$ and $\tau$. Said differently, the bulk acceleration horizon 
intersects the cylinder of any radius $\rho_{c}$, and imposing boundary conditions on the cylinder to define the global-CFT will always impose conditions on the metric for small $r_{\mathrm{R}}$ when $x_{\mathrm{R}}, t_{\mathrm{R}}$ are large enough. This behavior persists when $\rho_{c} \rightarrow \infty$. This is shown in Fig. 2(b). If, on the other hand, one wants to define the Rindler-CFT, then one needs to take large $r_{\mathrm{R} c}$ and permit arbitrarily large $x_{\mathrm{R}}$ and $t_{\mathrm{R}}$. Then one takes $r_{\mathrm{R} c} \rightarrow \infty$. This is clearly not consistent with the above procedure. This justifies our claim that the Rindler-CFT pair is different from the global-CFT.

\section{Global-CFT vs Rindler-CFT}

One may still wonder if the two CFTs are approximately the same in any sense. After all, when $\rho_{c}$ and $r_{\mathrm{R} c}$ are comparable, then one would expect the CFTs defined on the two surfaces to be related by conformal transformations. To analyze this, let us consider the global-CFT to see when it can be related to the Rindler-CFT by conformal transformations. In what follows we want $\rho$ to be large so we take $\rho \sim \mathcal{O}\left(\epsilon^{-1}\right)$ with $\epsilon \ll 1$. In this limit (13)-(15) become

$$
\begin{gathered}
r_{\mathrm{R}}^{2}=\rho^{2}\left(\sin ^{2} \phi-\sin ^{2} \tau\right)+\cos ^{2} \tau, \\
\tanh x_{\mathrm{R}}=-\left(1-\frac{1}{2 \rho^{2}}\right) \frac{\cos \phi}{\cos \tau}, \\
\tanh t_{\mathrm{R}}=\left(1+\frac{1}{2 \rho^{2}}\right) \frac{\sin \tau}{\sin \phi} .
\end{gathered}
$$

Equation (16) shows us that we have two distinct possibilities. One is when $\left(\sin ^{2} \phi-\sin ^{2} \tau\right) \sim \mathcal{O}(1)$ so that we have $r_{\mathrm{R}} \sim \rho \sim \mathcal{O}\left(\epsilon^{-1}\right)$ and the other is when $\left(\sin ^{2} \phi-\right.$ $\left.\sin ^{2} \tau\right) \sim \mathcal{O}\left(\epsilon^{2}\right)$ so that we have $r_{\mathrm{R}} \sim \mathcal{O}(1) \ll \rho$. We consider these two possibilities in detail.

Large $r_{\mathrm{R}}$ : For this limit we consider $\left(\sin ^{2} \phi-\sin ^{2} \tau\right) \sim$ $\mathcal{O}(1)$. We define $\hat{\phi}=\phi-\pi / 2$ and further consider the limit $\hat{\phi}, \tau \ll 1$. In this limit it is easy to see that we get

$$
\begin{gathered}
r_{\mathrm{R}}=\rho\left(1-\frac{1}{2}\left(\tau^{2}+\hat{\phi}^{2}\right)\right), \\
t_{\mathrm{R}}-x_{\mathrm{R}}=(\tau-\hat{\phi})+\frac{(\tau-\hat{\phi})^{3}}{6}+\frac{(\tau+\hat{\phi})}{2 \rho^{2}}, \\
t_{\mathrm{R}}+x_{\mathrm{R}}=(\tau+\hat{\phi})+\frac{(\tau+\hat{\phi})^{3}}{6}+\frac{(\tau-\hat{\phi})}{2 \rho^{2}} .
\end{gathered}
$$

Viewed as a diffeomorphism $\rho \rightarrow r_{\mathrm{R}}, \tau \rightarrow t_{\mathrm{R}}, \phi \rightarrow x_{\mathrm{R}}$, the above is realized as a Brown-Henneaux diffeomorphism (4)-(6) with $\xi^{ \pm}=-\frac{1}{6}(\tau \pm \phi)^{3}$. Note, one can take the limits $r_{\mathrm{R}} \rightarrow \infty$ and $\rho \rightarrow \infty$ together, and this means that the Fefferman-Graham expansions in

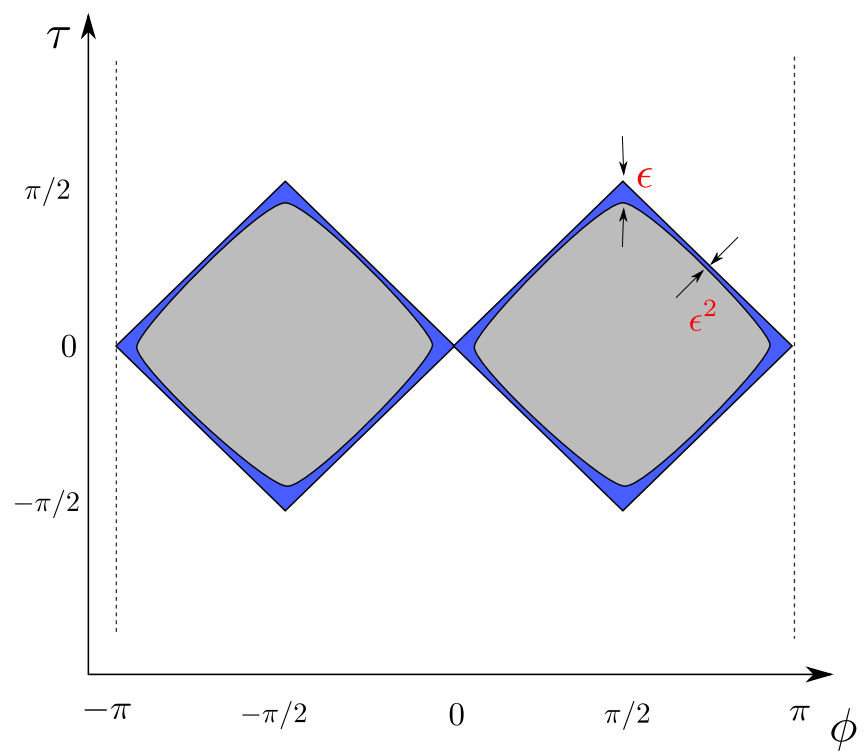

FIG. 3. We open up the global boundary cylinder for better visualization. The colored regions are the causal diamonds and are the interior of the curve the bulk Rindler-AdS horizons trace on the boundary cylinder. The global boundary cylinder is taken to be large with $\rho \sim \mathcal{O}\left(\epsilon^{-1}\right)$ and $\epsilon \ll 1$. The region where the Rindler-AdS radial coordinate $r_{\mathrm{R}} \sim \mathcal{O}(1)$ is shown in blue. This is the region where the Fefferman-Graham expansions in $\rho$ and $r_{\mathrm{R}}$ are not consistent and the Rindler-CFT cannot be approximated by the global-CFT.

the two radial coordinates are consistent. Writing the metric in the Fefferman-Graham form $(1)^{7}$ and using Brown-Henneaux diffeomorphisms, we get

$$
\begin{gathered}
\frac{d r_{\mathrm{R}}^{2}}{r_{\mathrm{R}}^{2}}+r_{\mathrm{R}}^{2}\left(-d t_{\mathrm{R}}^{2}+d x_{\mathrm{R}}^{2}\right)+\frac{1}{2}\left(d t_{\mathrm{R}}^{2}+d x_{\mathrm{R}}^{2}\right) \\
=\frac{d \rho^{2}}{\rho^{2}}+\rho^{2}\left(-d \tau^{2}+d \phi^{2}\right)-\frac{1}{2}\left(d \tau^{2}+d \phi^{2}\right)
\end{gathered}
$$

which shows that the negative Casimir energy vacuum state of the global-CFT appears to be an excited state of the Rindler-CFT.

Small $r_{\mathrm{R}}$ : For this limit we consider $\left(\sin ^{2} \phi-\sin ^{2} \tau\right) \lesssim$ $\mathcal{O}\left(\epsilon^{2}\right)$. This region is shown on the boundary cylinder in Fig. 3. To show that the Rindler-CFT and global-CFT are not conformally related, it suffices to show this in any one part of this region. We consider $\phi \sim \mathcal{O}(\epsilon)$ and $\tau \sim \mathcal{O}\left(\epsilon^{2}\right)$. The relations (13)-(15) become

$$
r_{\mathrm{R}}^{2}=\rho^{2} \phi^{2}+1 \sim \mathcal{O}(1),
$$

\footnotetext{
${ }^{7}$ As explained in footnote 6 this involves a redefinition of the radial coordinates for both foliations, but to avoid clutter we use the same labels.
} 


$$
\begin{gathered}
e^{2 x_{\mathrm{R}}}=\frac{1}{4}\left[\frac{1}{\rho^{2}}+\phi^{2}\right] \sim \mathcal{O}\left(\epsilon^{2}\right), \\
t_{\mathrm{R}}=\frac{\tau}{\phi}\left(1+\frac{1}{2 \rho^{2}}+\frac{1}{6} \phi^{2}\right) \sim \mathcal{O}(\epsilon) .
\end{gathered}
$$

Similarly, the relations (10)-(12) become

$$
\begin{gathered}
\rho=\frac{e^{-x_{\mathrm{R}}}}{2} r_{\mathrm{R}} \sim \mathcal{O}\left(\epsilon^{-1}\right), \\
\phi=2 \frac{\sqrt{r_{\mathrm{R}}^{2}-1}}{r_{\mathrm{R}}} e^{x_{\mathrm{R}}} \sim \mathcal{O}(\epsilon), \\
\tau=2 t \frac{\sqrt{r_{\mathrm{R}}^{2}-1}}{r_{\mathrm{R}}} e^{x_{\mathrm{R}}} \sim \mathcal{O}\left(\epsilon^{2}\right) .
\end{gathered}
$$

This does not have an interpretation as a small diffeomorphism and hence cannot be interpreted as a BrownHenneaux diffeomorphism. In addition, the coordinate $r_{\mathrm{R}}$ is now $\mathcal{O}(1)$ so it cannot be used as an expansion parameter for Brown-Henneaux diffeomorphisms or Fefferman-Graham expansions. Thus, now one cannot simultaneously take the limits $\rho \rightarrow \infty$ and $r_{\mathrm{R}} \rightarrow \infty$.

In summary, the two CFTs are approximated by each other when $r_{\mathrm{R}}$ scales as $\rho$, and this is in the vicinity of the center of the causal diamond. On the other hand, the two CFTs are distinct when $\rho$ is large but $r_{\mathrm{R}}$ is small, and this happens in the vicinity of the edges of the causal diamond. The width of the region in which $r_{\mathrm{R}}$ goes from $\mathcal{O}(\rho)$ to $\mathcal{O}(1)$ is proportional to the UV cutoff scale of the CFT.

\section{Global vs Poincaré boundary}

\section{Global and Poincaré coordinates and boundaries}

The story is analogous for the global vs Poincaré foliations. The metric in the Poincaré coordinates is

$$
d s^{2}=\frac{d r_{\mathrm{P}}^{2}}{r_{\mathrm{P}}^{2}}+r_{\mathrm{P}}^{2}\left(-d t_{\mathrm{P}}^{2}+d x_{\mathrm{P}}^{2}\right)
$$

where $r_{\mathrm{P}} \in(0, \infty)$ and $t_{\mathrm{P}}, x_{\mathrm{P}} \in(-\infty, \infty)$. There is a Cauchy ("Poincaré") horizon at $r_{\mathrm{P}}=0$. The relations between the global and Poincaré coordinates are

$$
\begin{gathered}
\rho=\frac{1}{2} r_{\mathrm{P}} \sqrt{\left[r_{\mathrm{P}}^{-2}+\left(-1+x_{\mathrm{P}}^{2}-t_{\mathrm{P}}^{2}\right)\right]^{2}+4 x_{\mathrm{P}}^{2}}, \\
\tan \tau=\frac{2 t_{\mathrm{P}}}{r_{\mathrm{P}}^{-2}+\left(1+x_{\mathrm{P}}^{2}-t_{\mathrm{P}}^{2}\right)}, \\
\tan \phi=-\frac{2 x_{\mathrm{P}}}{r_{\mathrm{P}}^{-2}+\left(-1+x_{\mathrm{P}}^{2}-t_{\mathrm{P}}^{2}\right)},
\end{gathered}
$$

and the inverse relations are

$$
\begin{gathered}
r_{\mathrm{P}}=\sqrt{1+\rho^{2}} \cos \tau+\rho \cos \phi, \\
t_{\mathrm{P}} r_{\mathrm{P}}=\sqrt{1+\rho^{2}} \sin \tau, \\
x_{\mathrm{P}} r_{\mathrm{P}}=\rho \sin \phi .
\end{gathered}
$$

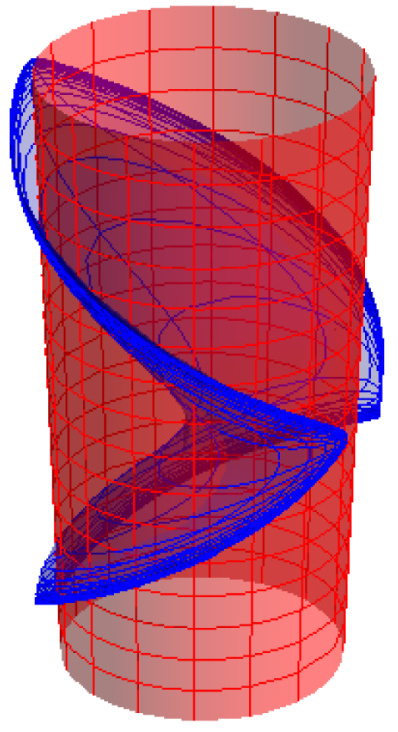

(a) Finite cutoff surfaces

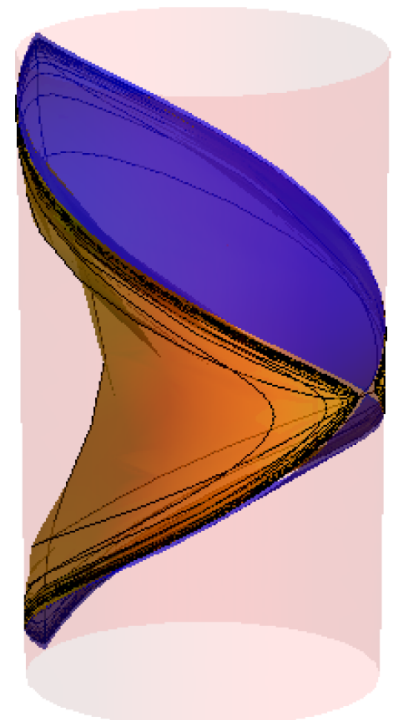

(b) Infinite cutoff surfaces

FIG. 4. (a) Global cutoff surface $\rho=\rho_{c}$ is shown in red and Poincaré cutoff surface $r_{\mathrm{P}}=r_{\mathrm{P} c}$ is shown in blue. (b) When we take $\rho_{c}$ to infinity, all the $r_{\mathrm{P}}$ surfaces bunch up along the edges of the causal diamond. Two of them are shown in the figure. 
The global-CFT is defined on the $\rho_{c} \rightarrow \infty$ surface, and the Poincare-CFT is defined on the $r_{\mathrm{P} c} \rightarrow \infty$ surface. In Fig. 4(a) we plot two such surfaces without taking the cutoff to infinity. In Fig. 4(b) we conformally compactify the global cylinder and see that surfaces of different constant $r_{\mathrm{P}}$ bunch up at the edges of the causal diamond. As in the Rindler-AdS case, imposing boundary conditions on the global boundary imposes conditions on small $r_{\mathrm{P}}$ surfaces also. This justifies our claim that Poincaré-CFT and global-CFT are different.

\section{Global-CFT vs Poincaré-CFT}

As before we would expect the global and the PoincaréCFTs to be approximately the same when $\rho_{c}$ and $r_{\mathrm{P} c}$ are comparable since then one would expect the CFTs defined on the two surfaces to be related by conformal transformations. To analyze this we will consider the globalCFT and try to see if and when it can be related to the Poincaré-CFT by conformal transformations. We want $\rho$ large so we take $\rho \sim \mathcal{O}\left(\epsilon^{-1}\right)$ with $\epsilon \ll 1$. In this limit (32)-(34) become

$$
\begin{gathered}
r_{\mathrm{P}}=\rho(\cos \tau+\cos \phi)+\cos \tau, \\
t_{\mathrm{P}}=\frac{\sin \tau}{\cos \tau+\cos \phi}\left(1+\frac{\cos \phi}{2 \rho^{2}(\cos \tau+\cos \phi)}\right), \\
x_{\mathrm{P}}=\frac{\sin \phi}{\cos \tau+\cos \phi}\left(1-\frac{\cos \tau}{2 \rho^{2}(\cos \tau+\cos \phi)}\right) .
\end{gathered}
$$

Equation (35) shows us that we have two distinct possibilities. The first is when $(\cos \phi+\cos \tau) \sim \mathcal{O}(1)$ so that we have $r_{\mathrm{P}} \sim \rho \sim \mathcal{O}\left(\epsilon^{-1}\right)$, and the other is when $(\cos \phi+$ $\cos \tau) \sim \mathcal{O}(\epsilon)$ so that we have $r_{\mathrm{R}} \sim \mathcal{O}(1) \ll \rho$. We consider these two possibilities in detail.

Large $r_{\mathrm{P}}$ : For this limit we consider $(\cos \phi+\cos \tau) \sim$ $\mathcal{O}(1)$. We further consider the limit $\phi, \tau \ll 1$. We get

$$
\begin{gathered}
r_{\mathrm{P}}=2 \rho\left(1-\frac{1}{4}\left(\tau^{2}+\phi^{2}\right)\right), \\
t_{\mathrm{P}}-x_{\mathrm{P}}=\frac{1}{2}(\tau-\phi)+\frac{(\tau-\phi)^{3}}{24}+\frac{(\tau+\phi)}{8 \rho^{2}}, \\
t_{\mathrm{P}}+x_{\mathrm{P}}=\frac{1}{2}(\tau+\phi)+\frac{(\tau+\phi)^{3}}{24}+\frac{(\tau-\phi)}{8 \rho^{2}} .
\end{gathered}
$$

Viewed as a diffeomorphism $\rho \rightarrow \frac{1}{2} r_{\mathrm{P}}, \tau \rightarrow \frac{1}{2} t_{\mathrm{P}}$, $\phi \rightarrow \frac{1}{2} x_{\mathrm{P}}$, the above is realized as a Brown-Henneaux diffeomorphism (4)-(6) with $\xi^{ \pm}=-\frac{1}{12}(\tau \pm \phi)^{3}$. One can take the limits $r_{\mathrm{P}} \rightarrow \infty$ and $\rho \rightarrow \infty$ together, and this means that the Fefferman-Graham expansions in the two radial coordinates are consistent. Writing the

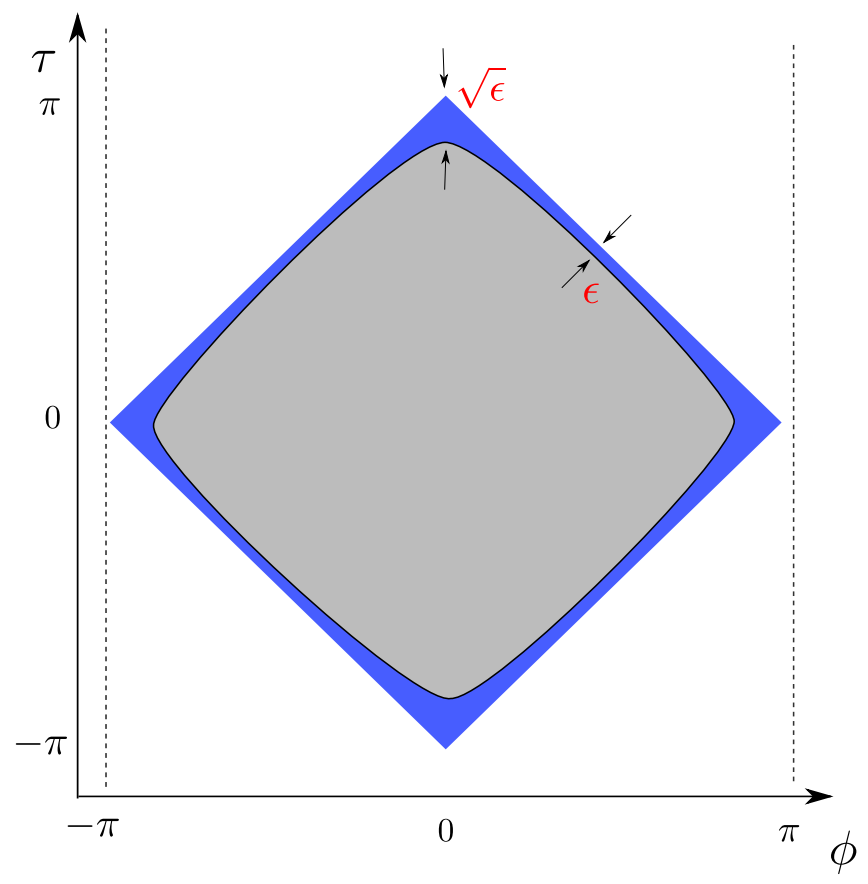

FIG. 5. We open up the boundary cylinder for better visualization. The colored region is the causal diamond and is the interior of the curve the bulk Poincaré horizon traces on the global boundary cylinder. The boundary cylinder is taken to be large with $\rho \sim \mathcal{O}\left(\epsilon^{-1}\right)$ and $\epsilon \ll 1$. The region where the Poincaré radial coordinate $r_{\mathrm{P}} \sim \mathcal{O}(1)$ is shown in blue. This is the region where the Fefferman-Graham expansions in $\rho$ and $r_{\mathrm{P}}$ are not consistent and the Poincaré-CFT cannot be approximated by the global-CFT.

metric in the Fefferman-Graham form $(1)^{8}$ and using Brown-Henneaux diffeomorphisms, we get

$$
\begin{aligned}
& \frac{d r_{\mathrm{P}}^{2}}{r_{\mathrm{P}}^{2}}+r_{\mathrm{P}}^{2}\left(-d t_{\mathrm{P}}^{2}+d x_{\mathrm{P}}^{2}\right) \\
& =\frac{d \rho^{2}}{\rho^{2}}+\rho^{2}\left(-d \tau^{2}+d \phi^{2}\right)-\frac{1}{2}\left(d \tau^{2}+d \phi^{2}\right)
\end{aligned}
$$

which shows that the negative Casimir energy vacuum state of the global-CFT appears to be the vacuum state of the Poincaré-CFT with zero energy.

Small $r_{\mathrm{P}}$ : For this limit we consider $(\cos \phi+\cos \tau) \lesssim \mathcal{O}(\epsilon)$. This region is shown on the boundary cylinder in Fig. 5. To show that the Poincaré-CFT and global-CFT are not conformally related it suffices to show this in any one part of this region. We define $\tilde{\phi} \equiv \pi-\phi$ and consider $\tilde{\phi} \sim$ $\mathcal{O}(\sqrt{\epsilon})$ and $\tau \sim \mathcal{O}(\epsilon)$. The relations (32)-(34) become

\footnotetext{
${ }^{8}$ As explained in footnote 6 this involves a redefinition of the global radial coordinate, but to avoid clutter we use the same label.
} 


$$
\begin{array}{r}
r_{\mathrm{P}}=\frac{1}{2} \rho \tilde{\phi}^{2}+1 \sim \mathcal{O}(1), \\
t_{\mathrm{P}}=\frac{2 \tau}{\tilde{\phi}^{2}} \sim \mathcal{O}(1), \\
x_{\mathrm{P}}=\frac{2}{\tilde{\phi}} \sim \mathcal{O}\left(\epsilon^{-1 / 2}\right) .
\end{array}
$$

Similarly, the relations (30) and (31) become

$$
\begin{gathered}
\rho=r_{\mathrm{P}} x_{\mathrm{P}}^{2} \sim \mathcal{O}\left(\epsilon^{-1}\right), \\
\tau=\frac{2 t_{\mathrm{P}}}{x_{\mathrm{P}}{ }^{2}} \sim \mathcal{O}(\epsilon), \\
\tilde{\phi}=\frac{2}{x_{\mathrm{P}}} \sim \mathcal{O}\left(\epsilon^{1 / 2}\right) .
\end{gathered}
$$

This does not have an interpretation as a small diffeomorphism and hence cannot be interpreted as a Brown-Henneaux diffeomorphism. Additionally, the coordinate $r_{\mathrm{P}}$ is now $\mathcal{O}(1)$ so it cannot be used as an expansion parameter for Brown-Henneaux diffeomorphisms or Fefferman-Graham expansions. Also, now one cannot simultaneously take the limits $\rho \rightarrow \infty$ and $r_{\mathrm{P}} \rightarrow \infty$.

As before, we see that the two CFTs are approximated by each other when $r_{\mathrm{P}}$ scales as $\rho$, which is in the vicinity of the center of the Poincare causal diamond. On the other hand, the two CFTs are distinct when $\rho$ is large but $r_{\mathrm{P}}$ is small, and this happens in the vicinity of the edges of the Poincare causal diamond. The width of the region in which

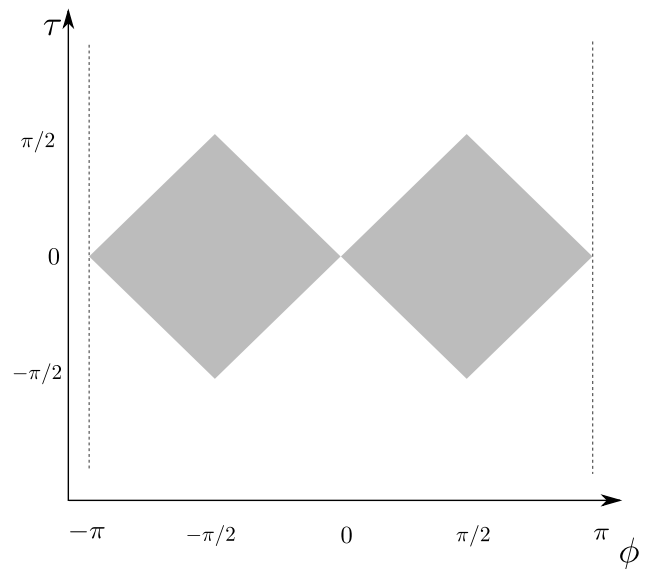

(a) Rindlerized-global-CFT
$r_{\mathrm{P}}$ goes from $\mathcal{O}(\rho)$ to $\mathcal{O}(1)$ is controlled by the UV cutoff scale of the CFT.

\section{CORRELATION FUNCTIONS IN GLOBAL-CFT, RINDLER-CFT, AND POINCARÉ-CFT}

\section{A. Differences between global-CFT and Rindler-CFT}

Consider the coordinate transformations

$$
\begin{gathered}
\tanh x_{\mathrm{R}}{ }^{\prime}=-\frac{\cos \phi}{\cos \tau}, \\
\tanh t_{\mathrm{R}}{ }^{\prime}=\frac{\sin \tau}{\sin \phi}
\end{gathered}
$$

This is a change of coordinates from the plane to a causal diamond that is the development of $\phi \in(0, \pi)$ in the cylinder. In Fig. 6(a) we plot the causal diamond (and also its antipodal version).

The two-point function of a primary operator $\mathcal{O}$ of weight $\Delta, \Delta$ on the cylinder is fixed by conformal invariance:

$$
\langle\mathcal{O}(\tau, \phi) \mathcal{O}(0,0)\rangle \sim\left(\frac{1}{\sin \left(\frac{\tau-\phi}{2}\right)} \frac{1}{\sin \left(\frac{\tau+\phi}{2}\right)}\right)^{2 \Delta}
$$

and under the conformal transformation (48) and (49), it becomes

$$
\left\langle\mathcal{O}\left(t_{\mathrm{R}}{ }^{\prime}, x_{\mathrm{R}}{ }^{\prime}\right) \mathcal{O}(0,0)\right\rangle \sim\left(\frac{1}{\sinh \left(\frac{t_{\mathrm{R}}{ }^{\prime}-x_{\mathrm{R}}{ }^{\prime}}{2}\right)} \frac{1}{\sinh \left(\frac{t_{\mathrm{R}}{ }^{\prime}+x_{\mathrm{R}}{ }^{\prime}}{2}\right)}\right)^{2 \Delta} .
$$

More general correlation functions inside the causal diamonds can be obtained from the ones on the cylinder, and

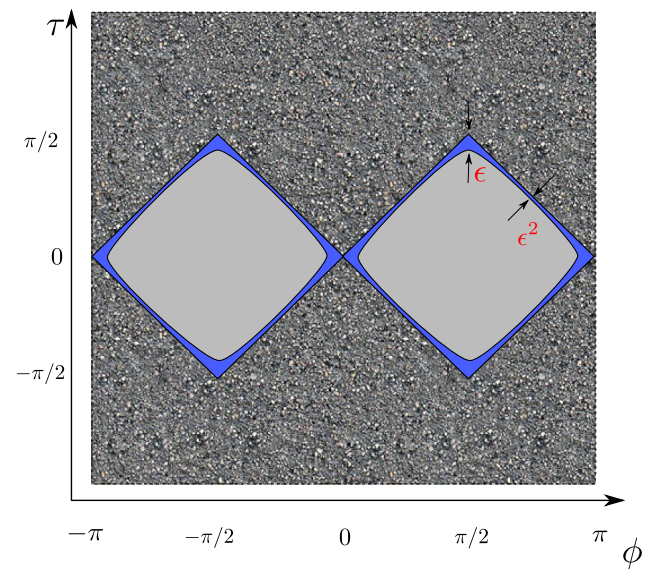

(b) Rindler-CFT

FIG. 6. We open up the boundary cylinder for better visualization. In (a) one formally divides the global-CFT into two halves and follows their causal development inside the causal diamonds. This is analogous to "Rindlerizing the global-CFT." This is not the same as the Rindler-CFT (b). While the two theories are approximately equal deep inside the diamonds, they start differing at the edges. The global-CFT is defined everywhere, but the Rindler-CFT is defined only inside the diamonds. Correlation functions of the latter do not give those of the former under analytic continuation. 
conversely the correlation functions inside a causal diamond give the correlation functions on the rest of the cylinder by analytic continuation.

The coordinate transformations (48) and (49) are analogous to the coordinate transformation to go from Minkowski spacetime to Rindler spacetime [31] and so, even though the CFT in the diamond is the same as the global-CFT, we refer to it as Rindlerized-global-CFT as it is restricted to the causal diamond.

The new coordinates $t_{\mathrm{R}}{ }^{\prime}, x_{\mathrm{R}}{ }^{\prime}$ on the global boundary cylinder can be related to the Rindler-AdS coordinates $t_{\mathrm{R}}, x_{\mathrm{R}}$ in the large $\rho$ limit using (17) and (18):

$$
\begin{aligned}
& \tanh x_{\mathrm{R}}=\tanh x_{\mathrm{R}}{ }^{\prime}\left(1-\frac{1}{2 \rho^{2}}\right), \\
& \tanh t_{\mathrm{R}}=\tanh t_{\mathrm{R}}{ }^{\prime}\left(1+\frac{1}{2 \rho^{2}}\right) .
\end{aligned}
$$

We can use these to understand what the correlators of the global-CFT imply for the correlators of the RindlerCFT. In the center of the causal diamonds $e^{-x_{\mathrm{R}}}, e^{-t_{\mathrm{R}}} \gg$ $\mathcal{O}(\epsilon)$ and we have $t_{\mathrm{R}}{ }^{\prime} \approx t_{\mathrm{R}}, x_{\mathrm{R}}{ }^{\prime} \approx x_{\mathrm{R}}$. The correlators of the Rindler-CFT can be approximated by

$$
\left\langle\mathcal{O}\left(t_{\mathrm{R}}, x_{\mathrm{R}}\right) \mathcal{O}(0,0)\right\rangle \approx\left(\frac{1}{\sinh \left(\frac{t_{\mathrm{R}}-x_{\mathrm{R}}}{2}\right)} \frac{1}{\sinh \left(\frac{t_{\mathrm{R}}+x_{\mathrm{R}}}{2}\right)}\right)^{2 \Delta} .
$$

However, when $e^{-x_{\mathrm{R}}}, e^{-t_{\mathrm{R}}} \sim \mathcal{O}(\epsilon)$ we get

$$
\begin{aligned}
& e^{-2 t_{\mathrm{R}}{ }^{\prime}}=e^{-2 t_{\mathrm{R}}}+\frac{1}{4 \rho^{2}}, \\
& e^{-2 x_{\mathrm{R}}{ }^{\prime}}=e^{-2 x_{\mathrm{R}}}-\frac{1}{4 \rho^{2}},
\end{aligned}
$$

and if we are considering the global-CFT, then for $e^{-x_{\mathrm{R}}{ }^{\prime}}$, $e^{-t_{\mathrm{R}}{ }^{\prime}} \sim \mathcal{O}(\epsilon)$ the correlators will continue to be given by (51) but in the same limit $e^{-x_{\mathrm{R}}}, e^{-t_{\mathrm{R}}} \sim \mathcal{O}(\epsilon)$, and the Rindler-CFT correlators will now deviate from the form (54). ${ }^{9}$

Let us try to understand what this means. If we were to consider the Rindler-CFT in a thermal state, then the correlators would be given by (54) for all values of $t_{\mathrm{R}}, x_{\mathrm{R}}$ since the two-point function in this case is fixed by conformal invariance. In other words, if we were to regulate the Rindler-CFT by putting some boundary conditions at $x_{\mathrm{R} \min }=-\tilde{x}$ and $x_{\mathrm{R} \max }=\tilde{x}$, then the correlators would be different for different boundary conditions but

\footnotetext{
${ }^{9}$ To be precise, since we are considering the CFT with a cutoff, there will be corrections of the form $\mathcal{O}\left(\left(t_{\mathrm{R}}{ }^{\prime} \pm x_{\mathrm{R}}{ }^{\prime}\right) / \rho\right)$ to $(51)$ and similar corrections to (54). These corrections are of a similar nature as the UV cutoff for both the CFTs are related. However, for the deviations of the Rindler-CFT correlators for large values of $x_{\mathrm{R}}, t_{\mathrm{R}}$ from (54), we see that the UV cutoff of the global-CFT induces an IR cutoff for the Rindler-CFT.
}

would all approach (54) in the limit $\tilde{x} \rightarrow \infty$ irrespective of the boundary conditions.

However, what we see above is that if we consider the global-CFT with a UV cutoff, then the Rindler-CFT correlators differ from (54) at the edges of the causal diamond. In the limit that the cutoff is pushed to infinity, the deviation from (54) happens at larger and larger values of $t_{\mathrm{R}}, x_{\mathrm{R}}$ but is always present.

Conversely, if we consider the Rindler-CFT then the correlators of the Rindlerized-global-CFT will differ from (51) at the edges of the causal diamond, and this immediately implies that the global-CFT correlators will not be the vacuum correlator (50). In fact, since the Rindler-CFT is not even defined outside the causal diamond, it seems likely that analytic continuation of correlators outside the causal diamonds will not work. This is shown in Fig. 6(b).

\section{B. Differences between global-CFT and Poincaré-CFT}

The analysis is similar to that in Sec. III A so we will be brief. Consider the coordinate transformations

$$
\begin{aligned}
t_{\mathrm{P}}{ }^{\prime} & =\frac{\sin \tau}{\cos \tau+\cos \phi}, \\
x_{\mathrm{P}}{ }^{\prime} & =\frac{\sin \phi}{\cos \tau+\cos \phi} .
\end{aligned}
$$

This is a change of coordinates from the plane to a causal diamond, which is the development of $\phi \in(0,2 \pi)$ in the global cylinder. We open up the cylinder and plot this causal diamond in Fig. 7(a).

Lucher and Mac [32] showed that correlation functions within this causal diamond can be analytically continued to the whole cylinder. This is because they are the same CFT related by coordinate transformations and so have the same dynamics. We refer to the CFT restricted inside this causal diamond as the Poincarized-global-CFT. The two-point correlator of a primary operator of weight $\Delta, \Delta$ inside the causal diamond is

$$
\left\langle\mathcal{O}\left(t_{\mathrm{P}}{ }^{\prime}, x_{\mathrm{P}}{ }^{\prime}\right) \mathcal{O}(0,0)\right\rangle \approx\left(\frac{1}{t_{\mathrm{P}}{ }^{2}-x_{\mathrm{P}}{ }^{\prime 2}}\right)^{2 \Delta} .
$$

The coordinates $t_{\mathrm{P}}{ }^{\prime}, x_{\mathrm{P}}{ }^{\prime}$ on the global boundary cylinder can be related to Poincaré coordinates $t_{\mathrm{P}}, x_{\mathrm{P}}$ in the large $\rho$ limit using (36) and (37):

$$
\begin{aligned}
& t_{\mathrm{P}}=t_{\mathrm{P}}{ }^{\prime}\left(1+\frac{1+t_{\mathrm{P}}{ }^{2}-x_{\mathrm{P}}{ }^{\prime 2}}{4 \rho^{2}}\right), \\
& x_{\mathrm{P}}=x_{\mathrm{P}}{ }^{\prime}\left(1-\frac{1-t_{\mathrm{P}}{ }^{2}+x_{\mathrm{P}}{ }^{2}}{4 \rho^{2}}\right) .
\end{aligned}
$$

We can use these to understand what the correlators of the global-CFT imply for the correlators of the Poincaré-CFT. When $t_{\mathrm{P}}, x_{\mathrm{P}} \sim \mathcal{O}(1)$ we have $t_{\mathrm{P}}{ }^{\prime} \approx t_{\mathrm{P}}, x_{\mathrm{P}}{ }^{\prime} \approx x_{\mathrm{P}}$. This is the 


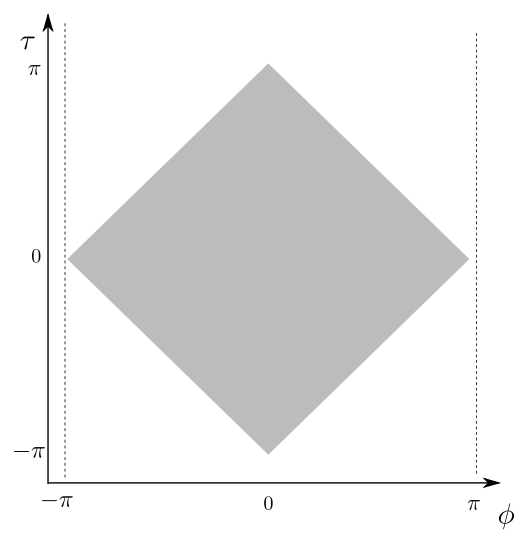

(a) Poincarized-global-CFT

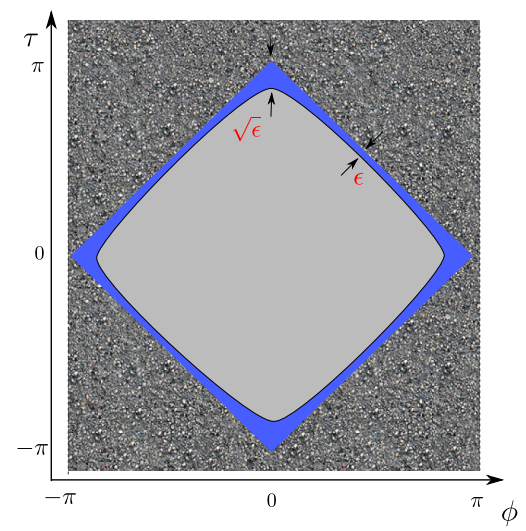

(b) Poincare-CFT

FIG. 7. We open up the boundary cylinder for better visualization. In (a) we show the causal development of the interval $(0,2 \pi)$. Correlation functions inside can be analytically continued to the full cylinder [32]. This is not the same as the Poincaré-CFT (b). While the two theories are approximately equal deep inside the diamond, they start differing at the edges. The global-CFT is defined everywhere but the Poincaré-CFT is defined only inside the diamond. Correlation functions of the latter do not give those of the former under analytic continuation.

center of the causal diamond, and the correlators of the Poincaré-CFT in this limit have the same expression as those of the Poincarized-global-CFT:

$$
\left\langle\mathcal{O}\left(t_{\mathrm{P}}, x_{\mathrm{P}}\right) \mathcal{O}(0,0)\right\rangle \approx\left(\frac{1}{t_{\mathrm{P}}^{2}-x_{\mathrm{P}}^{2}}\right)^{2 \Delta}
$$

However, when $t_{\mathrm{P}}, x_{\mathrm{P}} \sim \mathcal{O}\left(\epsilon^{-1}\right)$ the two start differing. If we are considering the global-CFT then for $t_{\mathrm{P}}{ }^{\prime}, x_{\mathrm{P}}{ }^{\prime} \sim$ $\mathcal{O}\left(\epsilon^{-1}\right)$ the correlators will continue to be given by (59). But in the same limit $t_{\mathrm{P}}, x_{\mathrm{P}} \sim \mathcal{O}\left(\epsilon^{-1}\right)$, and the PoincaréCFT correlators will now deviate from the form (62).

If we were in the vacuum state of the Poincare-CFT, the correlators would be given by (62) for all values of $t_{\mathrm{P}}, x_{\mathrm{P}}$ since this is determined by conformal invariance. In other words, if we put boundary conditions at $x_{\mathrm{Pmin}}=-\tilde{x}$ and $x_{\mathrm{Pmax}}=\tilde{x}$ the correlators would be sensitive to these boundary conditions, but in the limit $\tilde{x} \rightarrow \infty$ they would go to (62). We see precisely this kind of deviation for the Poincaré-CFT correlators when considering the globalCFT. Conversely, if we consider the Poincaré-CFT then the correlator of Poincarized-global-CFT will differ from (59) at the edges of the causal diamond, and this implies the global-CFT correlators will be different from (50). Since the Poincare-CFT is not even defined outside the causal diamond, it seems likely that analytic continuation of correlators outside the causal diamond will not work. This is shown in Fig. 7(b).

\section{Relating the CFTs}

We have argued that the various CFTs are different. An interesting question is whether it may be possible to view the Rindler-CFTs and the Poincaré-CFTs as deformations of the global-CFT. It seems the answer is yes.
From Sec. III A we see that the Rindlerized-global-CFTs and Rindler-CFTs are both defined on two copies of $R^{1,1}$. The former is in a particular entangled state (thermofield double state) by construction [31], and we can consider the latter in the same state. The two then appear to be the same but there is a subtle difference. In the case of the Rindlerized-global-CFT the Hilbert spaces associated with the two are the same as the ones associated with the intervals $(0, \pi)$ and $(\pi, 2 \pi)$ in global coordinates. These two Hilbert spaces are interacting (see Sec. II of [19]) across the points $\phi=0$ and $\phi=\pi$. This suggests that one may (roughly) think of the global-CFT as the pair of RindlerCFTs with an interaction between them across their respective boundaries. Similarly, one may (roughly) think of the global-CFT as the Poincare -CFT with an interaction across its two ends. We hope to make this rough picture more precise in the future.

\section{IMPLICATIONS FOR BULK PHYSICS}

\section{A. Conjecture for the bulk dual of Rindler-CFT and Poincaré-CFT}

We have established that correlation functions of Rindler-CFT, Poincaré-CFT, and global-CFT are different. Next we would like to understand the implications of this for the bulk physics. In general, local bulk physics is quite difficult to examine using the boundary field theory. However, global causal structures suggest some interesting new physics.

One might have expected that global AdS is dual to all these CFTs, but since their correlators are different, the bulk duals must be different too. Since the correlators are approximately equal until very close to the edges of the causal diamonds and since the causal diamonds are 
the projections of the event horizons, we conjecture that the bulk duals to the Rindler-CFT and the Poincaré-CFT will have a semiclassical description that matches that of the global-CFT until very close to the respective horizons and then start differing. The exact differences will depend on the boundary conditions on the Rindler-CFT and PoincaréCFT; we discuss two specific cases below.

\section{B. Implications for the massless BTZ black hole}

The massless BTZ black hole can be viewed as a quotient of $\mathrm{AdS}_{3}$ [33] that amounts to foliating in Poincaré coordinates and periodically identifying $x_{\mathrm{P}} \sim x_{\mathrm{P}}+2 \pi$. The question we want to ask is, what is the bulk dual when we periodically identify the $x_{\mathrm{P}}$ coordinate for the Poincaré-CFT? We refer to this as the periodically identified PIPC.

Let us begin by reviewing how the naive two-point functions of the PIPCs are obtained. Consider Euclidean $\mathrm{AdS}_{3}$ (see Appendix B for details). The boundary has the topology $S^{2}$. According to [23], in Poincare coordinates the boundary is at $r_{\mathrm{P}}=\infty$ (which has a topology $R^{2}$ ) with a point at $r_{\mathrm{P}}=0$ added. The boundary to the bulk propagator is given by

$$
\begin{aligned}
& K_{\mathrm{P}}^{\text {Euclidean }}\left(r_{\mathrm{P}}, x_{\mathrm{P}}, t_{\mathrm{E}, \mathrm{P}} ; r_{\mathrm{P}}{ }^{B}, x_{\mathrm{P}}{ }^{B}=0, t_{\mathrm{P}}{ }^{B}=0\right) \\
& \\
& \sim \begin{cases}r_{\mathrm{P}}^{-\Delta} & \text { when } r_{\mathrm{P}}{ }^{B}=0 \\
\left(\frac{r_{\mathrm{P}}}{1+r_{\mathrm{P}}^{2}\left[x_{\mathrm{P}}^{2}+t_{\mathrm{P}}^{2}\right]}\right)^{\Delta} & \text { when } r_{\mathrm{P}}{ }^{B}=\infty,\end{cases}
\end{aligned}
$$

where terms with superscripts denote boundary coordinates and $\Delta$ is interpreted as the conformal weight of the associated CFT operator. Naively, one obtains the boundary two-point function for the orbifolded geometry by summing over images of the Wick-rotated $r_{\mathrm{P}}{ }^{B}=\infty$ case of (63) and then using standard techniques to obtain

$$
\begin{aligned}
& \left\langle\mathcal{O}\left(t_{\mathrm{P}}, x_{\mathrm{P}}\right) \mathcal{O}(0,0)\right\rangle_{\text {PIPe }} \\
& \quad \sim \sum_{k=-\infty}^{\infty} \frac{1}{\left(x_{\mathrm{P}}+t_{\mathrm{P}}+2 \pi k\right)^{2 \Delta}\left(x_{\mathrm{P}}-t_{\mathrm{P}}+2 \pi k\right)^{2 \Delta}} .
\end{aligned}
$$

However, there is a problem with this procedure, and that is why we have crossed out the subscript on the correlator above. In Appendix B we explain that even in the Euclidean case the asymptotic limits of different foliations are not consistent. In particular, on a global $S^{2}$ of constant radius, large values of $x_{\mathrm{P}}$ correspond to small values of $r_{\mathrm{P}}{ }^{B}$. In the limit in which the global radius is taken to infinity, the transition region shrinks to zero in size and is captured by the "point at $r_{\mathrm{P}}=0$." For our purposes this implies that the $r_{\mathrm{P}}{ }^{B}=\infty$ case of (63) is only correct for $x_{\mathrm{P}}, t_{\mathrm{P}}$ values smaller than the UV cutoff scale. In the Wick-rotated case this means it is valid only inside the Poincare causal diamond away from the edges [see Fig. 7(b)]. Thus, the method of images can only be used as an approximation deep inside the causal diamond. Since the Poincaré-CFT and the global-CFT differ at the edges of the causal diamond and since under periodic identification the edges correspond to late times, the correct two-point function of PIPC would be approximated by (64) for early times but differ at late times. Consequently, according to our conjecture the bulk dual should resemble the massless BTZ until very close to the horizon but then should start differing. The length scale over which the transition takes place is governed by the UV cutoff.

Remarkably, we realize in hindsight that in the case of the D1-D5 system which flows in the IR to an $\mathcal{N}=(4,4)$ CFT, all this has been explicitly shown to be the case. Naively, the metric and dilaton of the D1-D5 system (with appropriate RR fluxes) are

$$
\begin{aligned}
d s_{\text {naive }}^{2}= & \frac{1}{\sqrt{g_{1} g_{5}}}\left(-d t_{\mathrm{P}}^{2}+d x_{\mathrm{P}}^{2}\right) \\
& +\sqrt{g_{1} g_{5}} \sum_{i=1}^{4} d x_{i}^{2}+\sqrt{\frac{g_{1}}{g_{5}} \sum_{i=1}^{4} d z_{i}^{2}} \\
e^{2 \phi}= & \frac{g_{1}}{g_{5}}, \quad g_{1}=1+\frac{Q_{1}}{r^{2}}, \quad g_{5}=1+\frac{Q_{5}}{r^{2}},
\end{aligned}
$$

where the charge radii are related to quantized charges by $Q_{1,5}=g l_{s}^{2} n_{1,5}$. The near-horizon limit of this geometry is massless $\mathrm{BTZ} \times T^{4} \times S^{3}$. So when the direction $x_{\mathrm{P}}$ is compactified the D1-D5 CFT plays the role of the PIPC.

However, the actual microstates of the D1-D5 system are not described by the massless BTZ. Instead they correspond to the Lunin-Mathur 2-charge fuzzball geometries [34-38]:

$$
\begin{aligned}
d s_{\text {string }}^{2}= & \frac{1}{\sqrt{\tilde{g}_{1} \tilde{g}_{5}}}\left(-\left(d t_{\mathrm{P}}-A_{i} d x^{i}\right)^{2}+\left(d x_{\mathrm{P}}+B_{i} d x^{i}\right)^{2}\right) \\
& +\sqrt{\tilde{g}_{1} \tilde{g}_{5}} \sum_{i=1}^{4} d x_{i}^{2}+\sqrt{\frac{\tilde{g}_{1}}{\tilde{g}_{5}}} \sum_{i=1}^{4} d z_{i}^{2}, \\
e^{2 \phi}= & \frac{\tilde{g}_{1}}{\tilde{g}_{5}}, \quad \tilde{g}_{5}(\vec{x})=1+\frac{Q_{5}}{L} \int_{0}^{L} \frac{d v}{|\vec{x}-\vec{F}(v)|^{2}}, \\
\tilde{g}_{1}(\vec{x})= & 1+\frac{Q_{5}}{L} \int_{0}^{L} \frac{|\dot{\vec{F}}(v)|^{2} d v}{|\vec{x}-\vec{F}(v)|^{2}}, \\
A_{i}(\vec{x})= & -\frac{Q_{5}}{L} \int_{0}^{L} \frac{\dot{F}_{i}(v) d v}{|\vec{x}-\vec{F}(v)|^{2}}, \quad d B=-\star_{4} d A,
\end{aligned}
$$

where $\star_{4}$ is taken with respect to the flat metric for the noncompact $x_{i}$ space and $v=x_{\mathrm{P}}-t_{\mathrm{P}}$. The solutions are governed by the profile function $\vec{F}(v)$. The length of integration is given by $L=2 \pi Q_{5}$ and one further has 


$$
Q_{1}=\frac{Q_{5}}{L} \int_{0}^{L} d v(\dot{F}(v))^{2} .
$$

For these solutions we see that if $|\vec{F}(v)|<b$, then at large distances, $r \gg b$, we recover the naive metric (65). Near $r \lesssim b$ metrics for different profile functions $\vec{F}(v)$ differ from each other.

The two-point functions in CFT states dual to these geometries have been worked out in the weak coupling limit [39]. For a subset of these states (conical-defect geometries $[40,41])$ we reproduce the result

$$
\begin{aligned}
& \left\langle\mathcal{O}\left(t_{\mathrm{P}}, x_{\mathrm{P}}\right) \mathcal{O}(0,0)\right\rangle_{\text {conical-defect }} \\
& \quad \sim \sum_{k=0}^{n-1} \frac{1}{\left(2 n \sin \frac{x_{\mathrm{P}}+t_{\mathrm{p}}+2 \pi k}{2 n}\right)^{2}\left(2 n \sin \frac{x_{\mathrm{P}}-t_{\mathrm{P}}+2 \pi k}{2 n}\right)^{2}}
\end{aligned}
$$

where $n$ is the order of the conical defect. In general, Ref. [39] found from studying the correlators that

"For large central charge (which leads to a good semiclassical limit), and sufficiently small time separation, a typical Ramond ground state of vanishing $R$-charge has the $M=0$ BTZ black hole as its effective description."

This is consistent with our claim that the true PoincaréCFT correlators agree with the global-CFT correlators deep inside the causal diamond which, after periodic identification, corresponds precisely to sufficiently small time separation). Reference [39] further states that

"At large time separation this effective description breaks down. The CFT correlators we compute take over, and give a response whose details depend on the microstate."

This, in particular, implies that none of the CFT correlators match the naive one (64) at large time separation. This is consistent with our claim that the correlators of the global-CFT and those of Poincaré-CFT disagree at the edges of the causal diamond (which after periodic identification just means large time separation).

Thus, in the case of massless BTZ embedded in type IIB supergravity compactified on $S^{1} \times T^{4}$ and $S^{1} \times K 3$, it is already known that the correct bulk dual to PIPC is not obtained from a simple orbifolding of $\mathrm{AdS}_{3}$. Instead the correct bulk duals of PIPC resemble the massless BTZ until very close to the horizon, and then quantum gravity effects modify the bulk and cut off the geometry outside the would-be horizons. This is shown in Fig. 8.

\section{Implications for the massive BTZ black hole}

The massive BTZ black hole can be viewed as a quotient of $\mathrm{AdS}_{3}$ [11] that amounts to foliating in Rindler-AdS coordinates and periodically identifying $x_{\mathrm{R}} \sim x_{\mathrm{R}}+2 \pi$. As before, the question we want to ask is, what is the bulk dual

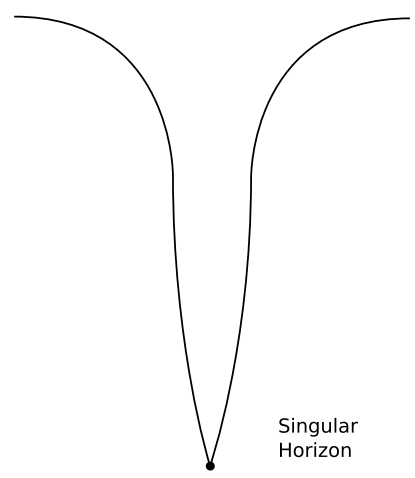

(a)

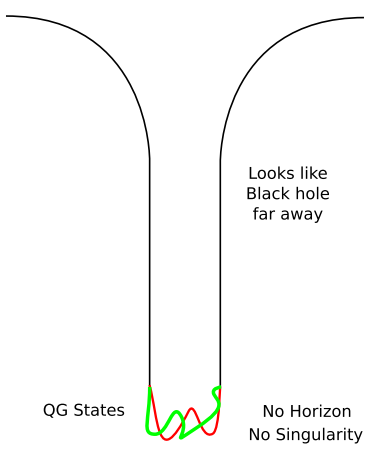

(b)
FIG. 8. (a) The geometry of the D1-D5 black hole has an outer flat space connected by a neck to the massless BTZ black hole. (b) The geometry of a generic state has outer flat space connected by a neck to a throat which ends in a smooth cap without horizons and singularities.

when we periodically identify the $x_{\mathrm{R}}$ coordinate for the Rindler-CFTs? We refer to these as the PIRCs.

The Carter-Penrose diagram of the massive BTZ is shown in Fig. 9(a). There are two regions outside the horizons which are asymptotically AdS. These regions have shared future (past) regions behind event horizons which end (begin) in a spacelike singularity. When viewed as an orbifold of global $\mathrm{AdS}_{3}$, the event horizons are the Rindler-AdS acceleration horizons and the singularities are orbifolding singularities.

In [14], Maldacena proposed that the massive BTZ black hole is dual to two decoupled CFTs which can be thought as living on the two boundaries (see Fig. 9) with topologies $R \times S^{1}$ and are in a particular entangled state called the thermofield double state (for details see [14]). In particular, the two CFTs are also supposed to capture the dynamics behind the horizons. This is a remarkable proposal: Imagine two excitations which we call boundary-Alice and boundary-Bob on either CFT. Since the CFTs are decoupled, these excitations have vanishing amplitude to interact. Yet their dual versions, bulk-Bob and bulk-Alice, have a nonzero amplitude to interact in the shared future region. While this may perhaps be possible if we consider a

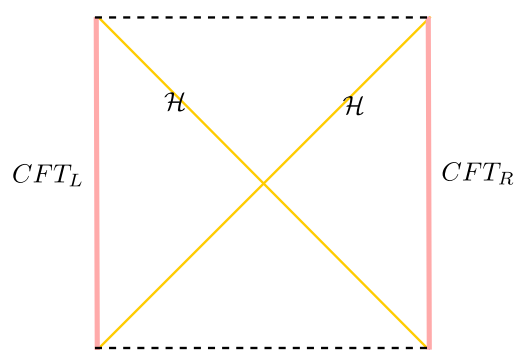

FIG. 9. The massive BTZ has two asymptotically AdS regions. These regions share the future and past regions behind the event horizons. 
$Z_{2}$ identification [42], with excitations also respecting the same identification, it seems hard to understand for the usual eternal BTZ black hole. Conceptual puzzles related to this issue have been raised in [18-20] and other puzzles with this picture have been raised in [16].

Nevertheless, one of the reasons to trust this picture has been that orbifolding in the bulk is innocuous at the horizons, even though its effects are felt inside at the orbifolding singularity. Thus, one hopes that the smoothness of horizons would be maintained. Further, it has been thought that the dual CFT to this orbifolded bulk can be described as quotients of the global-CFT [12-15]. One may then hope that the correlators from within the fundamental domain may be continued to the entire cylinder, and this could, in principle, be a dual description to dynamics behind the horizons. ${ }^{10}$

In these kinds of discussions it is either implicitly or explicitly assumed that the boundary CFT for studying Rindler-AdS and that for studying global AdS are the same (see Sec. II.4 of [43] and Sec. II.2 of [15] for instance). In this paper we have explicitly shown that this is not the case. Since the Rindler-AdS radial coordinate $r_{\mathrm{R}}$ becomes the radial coordinate of massive $\mathrm{BTZ}$ under orbifolding and the CFT dual to the BTZ is supposed to live on a surface of constant large radius, the CFT associated with the massive BTZ would be the Rindler-CFT instead of the global-CFT.

This difference is particularly relevant for the calculation of the CFT two-point function for the PIRCs. The naive two-point function for the periodically identified RindlerCFT is obtained in the following way. Reference [13] takes the Wick-rotated version of the $r_{\mathrm{P}}{ }^{B}=\infty$ case of (63) and uses the large $r_{\mathrm{R}}$ limit of the coordinate transformation between Poincaré and Rindler-AdS coordinates:

$$
\begin{gathered}
t_{\mathrm{P}} \pm x_{\mathrm{P}}=\frac{\sqrt{r_{\mathrm{R}}^{2}-1}}{r_{\mathrm{R}}} e^{t_{\mathrm{R}} \pm x_{\mathrm{R}}} \rightarrow e^{t_{\mathrm{R}} \pm x_{\mathrm{R}}}, \\
r_{\mathrm{P}}=r_{\mathrm{R}} e^{-x_{\mathrm{R}}}
\end{gathered}
$$

to get the bulk-boundary propagator in Rindler-AdS coordinates:

$$
\begin{gathered}
K_{\text {Rindler-Ads }}\left(r_{\mathrm{R}}, x_{\mathrm{R}}, t_{\mathrm{R}} ; r_{\mathrm{R}}{ }^{B}=\infty, x_{\mathrm{R}}{ }^{B}=0, t_{\mathrm{R}}{ }^{B}=0\right) \\
=\left(\frac{r_{\mathrm{R}}{ }^{-2} e^{-2 x_{\mathrm{R}}}}{\left[r_{\mathrm{R}}{ }^{-2}+\left(1-e^{-x_{\mathrm{R}}-t_{\mathrm{R}}}\right)\left(1-e^{-x_{\mathrm{R}}+t_{\mathrm{R}}}\right)\right]^{2}}\right)^{\Delta / 2} .
\end{gathered}
$$

To obtain the boundary two-point function from the orbifolded geometry, Ref. [13] sums over the images of (71) and then using standard techniques [23] obtains

\footnotetext{
${ }^{10}$ We thank Masaki Shigemori for discussions on this point.
}

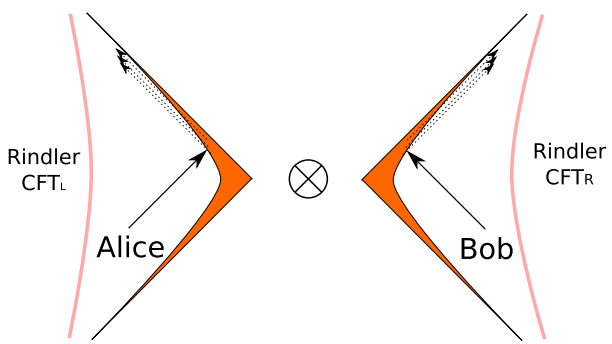

FIG. 10. According to the proposals in $[16,19,20]$ the bulk dual to two decoupled CFTs on $S^{1} \times R$ that are in the thermofield double state (which is equivalent to our PIRCs) are quantum geometries which resemble the massive BTZ at large distances but get capped outside the would-be horizons. Thus, there are no shared future or past regions. Our results are consistent with these proposals.

$$
\begin{aligned}
& \left\langle\mathcal{O}\left(x_{\mathrm{R}}, t_{\mathrm{R}}\right) \mathcal{O}(0,0)\right\rangle_{\text {PIRe }} \\
& \quad \sim \sum_{n=-\infty}^{\infty}\left(\frac{1}{\sinh \left(\frac{t_{\mathrm{R}}-x_{\mathrm{R}}-2 n \pi}{2}\right)} \frac{1}{\sinh \left(\frac{t_{\mathrm{R}}+x_{\mathrm{R}}+2 n \pi}{2}\right)}\right)^{2 \Delta} .
\end{aligned}
$$

Again, there is a problem with this procedure because we have crossed out the subscripts on (71) and (72). Not only is the Wick-rotated version of the $r_{\mathrm{P}}{ }^{B}=\infty$ case of (63) already an approximation for $x_{\mathrm{P}}, t_{\mathrm{P}}$ deep inside the Poincaré causal diamond (as explained in the previous section), but moreover, Ref. [13] further takes a large $r_{\mathrm{R}}$ limit which means we are restricting ourselves to $x_{\mathrm{R}}, t_{\mathrm{R}}$ deep inside the Rindler-AdS causal diamonds. One cannot then use the method of images on such an approximated propagator (71). Thus, the two-point function (72) is at best an approximation to the correct propagator for the PIRC for early times. This is good because the correlators (72) show a large time decay which is inconsistent with unitary CFTs $[14,22,44]$.

The correct two-point function of PIRC should resemble (72) until very close to the edge of the causal diamonds. After periodic identification, the edges correspond to late times. So consequently the two should agree at early times but not at late times. Consequently, according to our conjecture the bulk dual should resemble the massive BTZ until very close to the horizon and then start differing. The length scale over which the transition takes place is governed by the UV cutoff.

Our results support the conjecture forwarded by one of us in [19] (see also [16,20]), which states that the true bulk dual to the PIRCs is the one shown in Fig. 10 where the bulk dual resembles the massive BTZ outside the horizons, but there are no shared future and past regions. The spacetime caps off in "fuzzballs."11; This picture resolves the problems raised in $[16,18-20] .^{12}$

\footnotetext{
${ }^{11}$ For a review on fuzzballs see [45-50].

${ }^{12}$ For an alternate proposal for modifying the bulk see [22].
} 


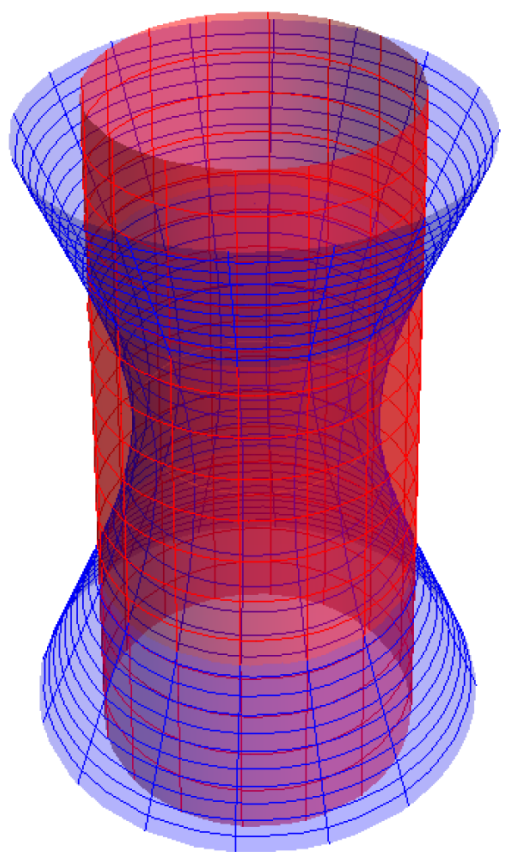

(a) Finite cutoff surfaces

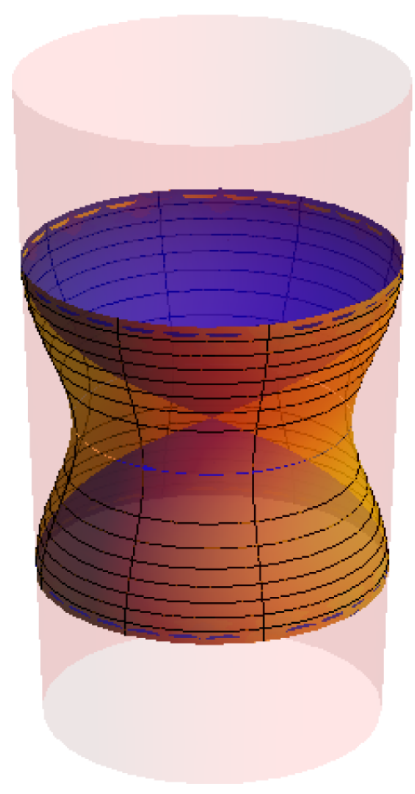

(b) Infinite cutoff surfaces

FIG. 11. (a) The global cutoff surface $\rho=\rho_{c}$ is shown in red, and the spherical Rindler-AdS cutoff surface $r_{\mathrm{SR}}=r_{\mathrm{SR} c}$ is shown in blue. (b) When we take $\rho_{c}$ to infinity, all the $r_{\mathrm{SR}}$ surfaces bunch up along the top and bottom edges of the "causal strip" which may be thought of as the union of causal diamonds for all the Rindler-AdS observers [51]. Two such constant $r_{\mathrm{SR}}$ surfaces are shown in the figure.

\section{ACKNOWLEDGMENTS}

It is a pleasure to thank Steve Avery, José Barbon, Vijay Balasubramanian, Saugata Chatterjee, Bartek Czech, Jan de Boer, Per Kraus, Gilad Lifschytz, Andrew Long, Gautam Mandal, Juan Maldacena, Samir Mathur, Shiraz Minwalla, Luboš Motl, Kyriakos Papadodimas, Joe Polchinski, Kalyana Rama, Suvrat Raju, Eray Sabancilar, Masaki Shigemori, Yogesh Srivastava, Nemani Suryanarayana, Tanmay Vachaspati, Mark Van Raamsdonk and Erik Verlinde for many useful discussions. We would also like to thank José Barbon, Vijay Balasubramanian, Bartek Czech, Per Kraus, Gilad Lifschytz, Juan Maldacena, Samir Mathur, Joe Polchinski, Mark Van Raamsdonk and Erik Verlinde for comments on an early draft of this paper. This work was supported in part by DOE Grant No. DE-FG02-09ER41624.

\section{APPENDIX A: GLOBAL BOUNDARY VS. SPHERICAL RINDLER BOUNDARY}

In this appendix we study one more foliation of global AdS which involves acceleration horizons in the bulk reaching out to the boundary-the spherical Rindler-AdS metric. ${ }^{13}$ The metric is

\footnotetext{
${ }^{13}$ Generalizations of this foliation were recently studied in [51], and similar considerations apply to those foliations also.
}

$d s^{2}=\frac{d r_{\mathrm{SR}}^{2}}{r_{\mathrm{SR}}^{2}-1}+\left(r_{\mathrm{SR}}^{2}-1\right)\left(-d t_{\mathrm{SR}}^{2}+\cosh ^{2} t_{\mathrm{SR}} d \phi^{2}\right)$,

where $r_{\mathrm{SR}} \in(1, \infty), t_{\mathrm{SR}} \in(-\infty, \infty)$ and $\phi \sim \phi+2 \pi$. The relation between the spherical Rindler-AdS and global coordinates is

$$
\begin{gathered}
\rho=\sqrt{r_{\mathrm{SR}}^{2}-1} \cosh t_{\mathrm{SR}}, \\
\cot \tau=\frac{r_{\mathrm{SR}}}{\sqrt{r_{\mathrm{SR}}^{2}-1}} \frac{1}{\sinh t_{\mathrm{SR}}},
\end{gathered}
$$

and the $\phi$ coordinate is the same. The inverse relations are

$$
\begin{gathered}
r_{\mathrm{SR}}=\cosh \rho \cos \tau \\
\sinh t_{\mathrm{SR}}=\frac{\cosh \rho \sin \tau}{\sqrt{\cosh ^{2} \rho \cos ^{2} \tau-1}} .
\end{gathered}
$$

We see the same theme as before. We can define the globalCFT by fixing boundary conditions on $\rho_{c}$. However, for large enough $t_{\mathrm{SR}}$, we get a $r_{\mathrm{SR}}=1$ surface (and all other small $r_{\mathrm{SR}}$ surfaces) intersecting the global cylinder (see Fig. 11). Imposing boundary conditions on the large $\rho_{c}$ surface puts conditions on small $r_{\mathrm{SR}}$ surfaces also. Different boundary conditions suggest that bulk physics would also be different close to the horizon scale. It is possible that the 
de Sitter CFT dual to spherical Rindler-AdS may be viewable as a deformation of the global-CFT. We hope to come back to this issue in the future.

\section{APPENDIX B: EUCLIDEAN VERSION}

Euclidean $\mathrm{AdS}_{d+1}$ is a ball with the boundary having the topology of $S^{d}$. Specializing to $\mathrm{AdS}_{3}$ we can write the metric in coordinates which enjoy the symmetries of Euclidean AdS:

$$
d s^{2}=\frac{d \tilde{\rho}^{2}}{\tilde{\rho}^{2}+1}+\tilde{\rho}^{2}\left(d \tilde{\theta}^{2}+\sin ^{2} \tilde{\theta} d \tilde{\phi}^{2}\right)
$$

We can also use Poincaré coordinates (29) with Poincaré time Wick rotated:

$$
d s^{2}=\frac{d r_{\mathrm{P}}^{2}}{r_{\mathrm{P}}^{2}}+r_{\mathrm{P}}^{2}\left(d t_{\mathrm{P}, \mathrm{E}}^{2}+d x_{\mathrm{P}}^{2}\right)
$$

One can also foliate $\mathrm{AdS}_{3}$ by Wick rotating the global time for global coordinates or the Rindler-AdS time for RindlerAdS coordinates. However, for our purpose the above two suffice.

The relations between these coordinates are

$$
\begin{gathered}
\sqrt{\tilde{\rho}^{2}+1}=\frac{1}{2 r_{\mathrm{P}}}\left(1+r_{\mathrm{P}}^{2}\left(1+x_{\mathrm{P}}^{2}+t_{\mathrm{P}, \mathrm{E}}^{2}\right)\right), \\
\tan \tilde{\phi}=\frac{t}{x} \\
\cos \tilde{\theta}=\frac{\frac{1}{2 r_{\mathrm{P}}}\left(1-r_{\mathrm{P}}^{2}\left(1-x_{\mathrm{P}}^{2}-t_{\mathrm{P}, \mathrm{E}}^{2}\right)\right)}{\tilde{\rho}}
\end{gathered}
$$

and the inverse relations are

$$
\begin{gathered}
r_{\mathrm{P}}=\sqrt{\tilde{\rho}^{2}+1}-\tilde{\rho} \cos \tilde{\theta}, \\
x_{\mathrm{P}} r_{\mathrm{P}}=\tilde{\rho} \sin \tilde{\theta} \cos \tilde{\phi}, \\
t_{\mathrm{P}, \mathrm{E}} r_{\mathrm{P}}=\tilde{\rho} \sin \tilde{\theta} \sin \tilde{\phi} .
\end{gathered}
$$

The large $\tilde{\rho}$ limits of (B6)-(B8) are

$$
r_{\mathrm{P}}=2 \sin ^{2}(\tilde{\theta} / 2) \tilde{\rho},
$$

$$
x_{\mathrm{P}}=\cot (\theta / 2) \cos \tilde{\phi}\left(1-\frac{1}{4 \sin ^{2}(\tilde{\theta} / 2) \tilde{\rho}^{2}}\right),
$$

$$
t_{\mathrm{P}, \mathrm{E}}=\cot (\theta / 2) \sin \tilde{\phi}\left(1-\frac{1}{4 \sin ^{2}(\tilde{\theta} / 2) \tilde{\rho}^{2}}\right) .
$$

If we take a large sphere at $\tilde{\rho} \sim \mathcal{O}\left(\epsilon^{-1}\right)$ with $\epsilon \ll 1$ then we have two cases. One has $\tilde{\theta} \sim \mathcal{O}(1)$ for which $r_{\mathrm{P}} \sim \tilde{\rho}$, and the other has $\tilde{\theta} \sim \mathcal{O}\left(\epsilon^{1 / 2}\right)$ for which $r_{\mathrm{P}} \sim \mathcal{O}(1)$. In the latter regime $x_{\mathrm{P}}^{2}+t_{\mathrm{P}, \mathrm{E}}{ }^{2} \sim \mathcal{O}\left(\epsilon^{-1}\right)$. In the limit that the UV cutoff is taken to infinity one can think of the boundary of Euclidean $\mathrm{AdS}_{3}$ being at $r_{\mathrm{P}}=\infty$ with "a point added at zero" [23]. However, as in the Lorentzian case, for any large but finite $\tilde{\rho}$, surfaces of arbitrarily small $r_{\mathrm{P}}$ intersect the surface of constant $\tilde{\rho}$.
[1] V. Balasubramanian and P. Kraus, A stress tensor for anti-de Sitter gravity, Commun. Math. Phys. 208, 413 (1999).

[2] V. Balasubramanian, P. Kraus, and A. E. Lawrence, Bulk versus boundary dynamics in anti-de Sitter space-time, Phys. Rev. D 59, 046003 (1999).

[3] G. Compere and D. Marolf, Setting the boundary free in AdS/CFT, Classical Quantum Gravity 25, 195014 (2008).

[4] G. Compère, W. Song, and A. Strominger, New boundary conditions for $\mathrm{AdS}_{3}$, J. High Energy Phys. 05 (2013) 152.

[5] S. G. Avery, R. R. Poojary, and N. V. Suryanarayana, An $\mathrm{sl}(2, \mathbb{R})$ current algebra from $\mathrm{AdS}_{3}$ gravity, J. High Energy Phys. 01 (2014) 144.

[6] R. Emparan, C. V. Johnson, and R. C. Myers, Surface terms as counterterms in the AdS/CFT correspondence, Phys. Rev. D 60, 104001 (1999).
[7] B. D. Chowdhury, Limitations of holography, Int. J. Mod. Phys. D 24, 1550008 (2015).

[8] C. A. Ballón Bayona and N. R. F. Braga, Anti-de Sitter boundary in Poincaré coordinates, Gen. Relat. Gravit. 39, 1367 (2007).

[9] C. Fefferman and C. Graham, Conformal invariants, Elie Cartan et les Math ematiques d'aujourd'hui, Astérisque 95-116 (1985).

[10] M. Banados, C. Teitelboim, and J. Zanelli, The Black Hole in Three-Dimensional Space-Time, Phys. Rev. Lett. 69, 1849 (1992).

[11] M. Banados, M. Henneaux, C. Teitelboim, and J. Zanelli, Geometry of the $(2+1)$ black hole, Phys. Rev. D 48, 1506 (1993).

[12] G. T. Horowitz and D. Marolf, A new approach to string cosmology, J. High Energy Phys. 07 (1998) 014. 
[13] E. Keski-Vakkuri, Bulk and boundary dynamics in BTZ black holes, Phys. Rev. D 59, 104001 (1999).

[14] J. M. Maldacena, Eternal black holes in anti-de Sitter, J. High Energy Phys. 04 (2003) 021.

[15] S. Hemming, E. Keski-Vakkuri, and P. Kraus, Strings in the extended BTZ space-time, J. High Energy Phys. 10 (2002) 006.

[16] B. S. Kay, Instability of enclosed horizons, Gen. Relativ. Gravit. 46, 1727 (2014).

[17] V. Balasubramanian, P. Kraus, A. E. Lawrence, and S. P. Trivedi, Holographic probes of anti-de Sitter space-times, Phys. Rev. D 59, 104021 (1999).

[18] D. Marolf and A. C. Wall, Eternal black holes and superselection in AdS/CFT, Classical Quantum Gravity 30, 025001 (2013).

[19] S. G. Avery and B. D. Chowdhury, No holography for eternal AdS black holes, arXiv:1312.3346.

[20] S. D. Mathur, What is the dual of two entangled CFTs?, arXiv:1402.6378

[21] S. Hawking, Information preservation and weather forecasting for black holes, arXiv:1401.5761.

[22] D. Kabat and G. Lifschytz, Finite $n$ and the failure of bulk locality: Black holes in AdS/CFT, J. High Energy Phys. 09 (2014) 077.

[23] E. Witten, Anti-de Sitter space and holography, Adv. Theor. Math. Phys. 2, 253 (1998).

[24] J. D. Brown and M. Henneaux, Central charges in the canonical realization of asymptotic symmetries: An example from three-dimensional gravity, Commun. Math. Phys. 104, 207 (1986).

[25] R. Emparan, AdS/CFT duals of topological black holes and the entropy of zero energy states, J. High Energy Phys. 06 (1999) 036.

[26] A. Hamilton, D. N. Kabat, G. Lifschytz, and D. A. Lowe, Holographic representation of local bulk operators, Phys. Rev. D 74, 066009 (2006).

[27] R. C. Myers and A. Sinha, Seeing a c-theorem with holography, Phys. Rev. D 82, 046006 (2010).

[28] M. Parikh and P. Samantray, Rindler-AdS/CFT, arXiv: 1211.7370 .

[29] M. Parikh, P. Samantray, and E. Verlinde, Rotating RindlerAdS Space, Phys. Rev. D 86, 024005 (2012).

[30] J. L. Barbón and E. Rabinovici, Conformal complementarity maps, J. High Energy Phys. 12 (2013) 023.

[31] H. Casini, M. Huerta, and R. C. Myers, Towards a derivation of holographic entanglement entropy, J. High Energy Phys. 05 (2011) 036.

[32] M. Lüscher and G. Mack, Global conformal invariance in quantum field theory, Commun. Math. Phys. 41, 203 (1975).
[33] J. Parsons and S. F. Ross, Strings in extremal BTZ black holes, J. High Energy Phys. 04 (2009) 134.

[34] O. Lunin and S. D. Mathur, Statistical Interpretation of Bekenstein Entropy for Systems with a Stretched Horizon, Phys. Rev. Lett. 88, 211303 (2002).

[35] O. Lunin, J. M. Maldacena, and L. Maoz, Gravity solutions for the D1-D5 system with angular momentum, arXiv: hep-th/0212210.

[36] O. Lunin and S. D. Mathur, Metric of the multiply wound rotating string, Nucl. Phys. B610, 49 (2001).

[37] K. Skenderis and M. Taylor, Fuzzball Solutions and D1-D5 Microstates, Phys. Rev. Lett. 98, 071601 (2007).

[38] I. Kanitscheider, K. Skenderis, and M. Taylor, Fuzzballs with internal excitations, J. High Energy Phys. 06 (2007) 056.

[39] V. Balasubramanian, P. Kraus, and M. Shigemori, Massless black holes and black rings as effective geometries of the D1-D5 system, Classical Quantum Gravity 22, 4803 (2005).

[40] J. M. Maldacena and L. Maoz, Desingularization by rotation, J. High Energy Phys. 12 (2002) 055.

[41] V. Balasubramanian, J. de Boer, E. Keski-Vakkuri, and S. F. Ross, Supersymmetric conical defects: Towards a string theoretic description of black hole formation, Phys. Rev. D 64, 064011 (2001).

[42] M. K. Parikh, I. Savonije, and E. P. Verlinde, Elliptic de Sitter space: dS/Z(2), Phys. Rev. D 67, 064005 (2003).

[43] J. M. Maldacena and A. Strominger, AdS(3) black holes and a stringy exclusion principle, J. High Energy Phys. 12 (1998) 005.

[44] J. Barbon and E. Rabinovici, Long time scales and eternal black holes, Fortschr. Phys. 52, 642 (2004).

[45] I. Bena and N. P. Warner, One ring to rule them all...and in the darkness bind them?, Adv. Theor. Math. Phys. 9, 667 (2005).

[46] S. D. Mathur, The fuzzball proposal for black holes: An elementary review, Fortschr. Phys. 53, 793 (2005).

[47] K. Skenderis and M. Taylor, The fuzzball proposal for black holes, Phys. Rep. 467, 117 (2008).

[48] V. Balasubramanian, J. de Boer, S. El-Showk, and I. Messamah, Black holes as effective geometries, Classical Quantum Gravity 25, 214004 (2008).

[49] B. D. Chowdhury and A. Virmani, Modave lectures on fuzzballs and emission from the D1-D5 system, arXiv: 1001.1444

[50] I. Bena and N. P. Warner, Resolving the structure of black holes: Philosophizing with a hammer, arXiv:1311.4538.

[51] V. Balasubramanian, B. D. Chowdhury, B. Czech, J. de Boer, and M.P. Heller, A hole-ographic spacetime, Phys. Rev. D 89, 086004 (2014). 